\title{
INFLUENCING OF THE PILE-UP AND THE SINK-IN BY THE COEFFICIENT OF FRICTION IN THE NANOINDENTATION TEST
}

\author{
Kovář J.*, Fuis V.** Čtvrtlík R.***
}

\begin{abstract}
The influence of the coefficient of friction between an indenter and a specimen on the height of the pile-up or depth of the sink-in during the nanoindentation test is discussed in this paper. When the indenter is pressed into the hard material, the sink-in behavior causes the bending of the specimen's surface under the indenter. When the indenter is pressed into the soft material, the deformed material accumulates under the sides of the indenter and makes a pile-up region. The pile-up or sink-in behavior influences the contact area. In this paper, the finite element method was used for calculation of the dependency between the depth of the sink-in or height of the pile-up and the coefficient of friction. The results showed that while there is not any dependency between the friction and the depth of the sink-in, there is a strong dependency between the friction and the height of the pile-up until a specific value of the coefficient of friction. When the pile-up behavior occurs, the friction influences the contact area and should be included into the calculation of the contact area.
\end{abstract}

\section{Keywords: Nanoindentation, Pile-up, Sink-in, Coefficient of friction, FEM.}

\section{Introduction}

Nanoindentation test is often used for measuring of the surface's material parameters which cannot be measured by conventional macro tests. During the test, a diamond indenter of known geometry is pressed into the material and the indentation curve, which is the dependency of the force on the displacement of the indenter, is measured. The displacement of the indenter is usually in the range from a few tens to a few hundreds of nanometers.

When the Berkovich indenter is pressed into the material, the surface of the specimen can behave in two ways. The first case is the sink-in effect (Fig. 1a), when the surface of the material bends inwards and downwards under the indenter. The other case occurs, if the plastic deformation is large and the indented material flows upwards along the sides of the indenter, accumulates and forms the pile-up (Fig. 1b). These effects are most pronounced for the central parts of the indenter's faces and declines towards the indenter edges, where nearly no influence occurs. It should be noted that for purely elastic indentations only sink-in can occur. It is well known that parameters governing the amount of sink-in and pile-up are Young's modulus, yield stress and strain-hardening properties.
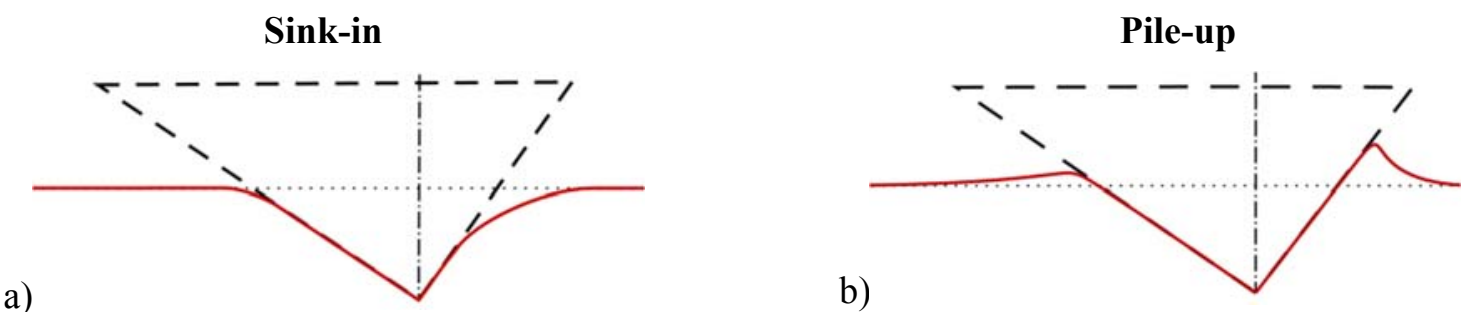

Fig. 1: Sink-in and pile-up effect.

\footnotetext{
* Ing. Jaroslav Kovár: Institute of Solid Mechanics, Mechatronics and Biomechanics, Brno University of Technology, Technická 2896/2; 616 69, Brno; CZ, Jaroslav.Kovar@vut.cz

** Assoc. Prof. Ing. Vladimír Fuis, PhD.: Centre of Mechatronics - Institute of Thermomechanics of the Czech Academy of Sciences - branch Brno and Faculty of Mechanical Engineering, Brno University of Technology, Technická 2896/2; 619 69, Brno, CZ, fuis@it.cas.cz

*** Mgr. Radim Čtvrtlík, PhD.: Institute of Physics of the Czech Academy of Sciences, Joint Laboratory of Optics of Palacký, University and Institute of Physics of the AS CR; 17. listopadu 50a; 772 07, Olomouc, CZ, ctvrtlik@fzu.cz
} 
With the use of the Oliver-Pharr analysis (Oliver, 1992), the indentation elastic modulus that might be under specific circumstances directly related to Young's modulus can be determined from the unloading part of the indentation curve and analysis of the contact region. However, this analysis is based on the assumption that the contact area is determined correctly. When the pile-up occurs, the contact area is bigger than it is expected in the Oliver-Pharr analysis (Oliver, 2004) and appropriate corrections must be applied.

The friction between the indenter and the specimen could have an influence on the pile-up or sink-in behaviour. In this paper, FEM simulations of the nanoindentation test will be made to calculate the indentation curves, which will be compared with the experiment, then the dependencies between the height of the pile-up and the coefficient of friction, and the depth of the sink-in and the coefficient of friction will be determined and the impact of the friction will be assessed.

\section{Methods}

The FEM was chosen for calculations because it allows the calculation of the deformation and stresses field in the specimen beneath the penetrating indenter. The tested specimen was assumed as a cylindrical body and then, because the Berkovich indenter has three planes of symmetry, only one sixth of the geometry was modelled. The dimensions of the specimen and the indenter were made sufficiently big to eliminate the influence of the edges on the results (Fig. 2). The contact region was made between the indenter and the specimen. The surface of the indenter was chosen as the TARGET area and surface of the specimen was chosen as the CONTACT area. To obtain more precise results, the tip and the edges near the indenter were blunted. Near the blunted tip, new blunted edges were created to remove sharp edges. The radius of the bluntness was chosen to be $400 \mathrm{~nm}$ (Kovář et al., 2019).

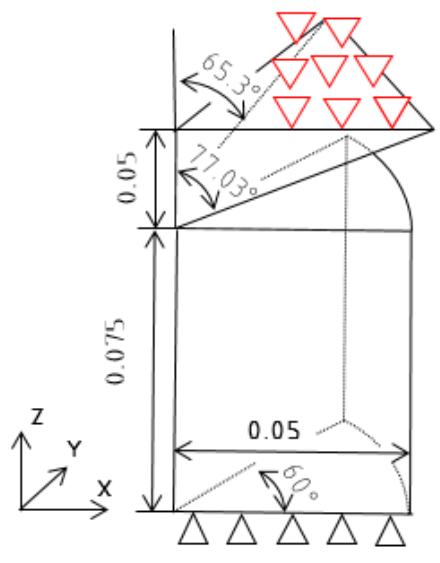

Fig. 2: Geometry of the tested specimen [mm].

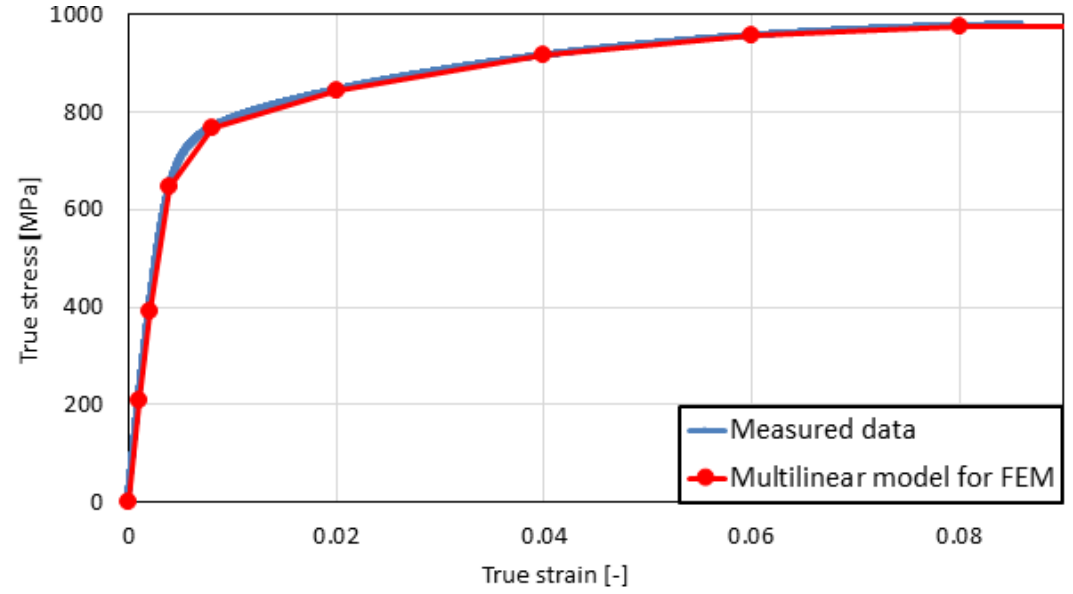

Fig. 3: Comparing of the measured data and multilinear model used for the FEM.

To obtain the sink-in and the pile-up behavior, two distinct materials of specimens were considered. The first one was from fused silica, modelled as an ideal elasto-plastic material with the Young's modulus $\mathrm{E}_{\mathrm{fs}}=72 \mathrm{GPa}$ and Poisson's ratio $\mu_{\mathrm{fs}}=0.17$ (Kovár, 2019) Plasticity was defined with the commonly used yield stress $\mathrm{R}_{\mathrm{e}-\mathrm{fs}}=6 \mathrm{GPa}$. The second one was specimen form the $\mathrm{X} 5 \mathrm{CrNiCuNb} 16-4$ steel which was modelled as multilinear material with the elastic properties $E_{\mathrm{s}}=210 \mathrm{GPa}$ and $\mu_{\mathrm{s}}=0.33$. Parameters for the multilinear model were obtained from the tensile test, which was made by the Institute of Materials, BUT. The multilinear model accurately described the measured stress-strain curve (Fig. 3). The diamond indenter was modelled as linear elastic material with the following material properties: $E_{d}=1141 \mathrm{GPa}, \mu_{d}=0.07$ (Kovár, 2019). This model of material assumed that there is no plastic deformation of the indenter.

After defining the model of material, boundary conditions were applied to the model. On the bottom area of the specimen, the displacement in the direction of the $\mathrm{Z}$ axis was fixed. Other boundary conditions were made from the conditions of symmetry because only one sixth of the geometry was modelled. Displacements perpendicular to the planes of symmetry were fixed on these planes.

In order to achieve a better convergence of the task, deformational loading was used. In the first loadstep, the specimen was loaded with the displacement of the upper area of the indenter by $500 \mathrm{~nm}$ plus the gap between the indenter and the specimen caused by the bluntness. In the second loadstep, unloading occurred. 
The mesh for FEM (Fig. 4) was created from SOLID 185 elements. In the contact area, a fine mesh was created. Between the parts with fine and coarse mesh, the transitional area from tetraedrical elements was made. Tetraedrical elements were used in the volume with small stress gradients and then their inaccuracy should be small. In the contact region, the CONTA 174 / TARGE 170 elements were used.
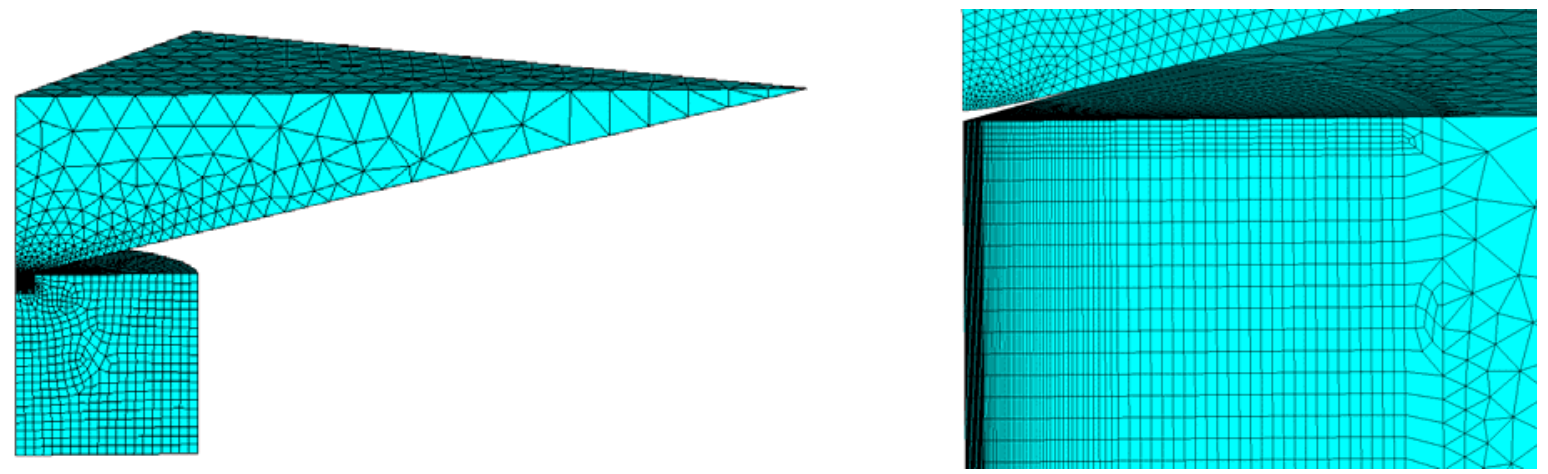

Fig. 4: Mesh used for FEM and the detailed view of the fine mesh in the contact area (right).

Since the FEM calculation had involved three nonlinearities (contact, high strains, and plasticity), special options in the calculation had to be done. Large displacements were assumed and each loadstep was divided into more than 100 substeps. To obtain the dependency between the height of the pile-up and the coefficient of friction, several calculations with different values of the coefficient of friction were done.

After the FEM calculations, the resultant forces on the bottom area of the specimen were calculated and due to the symmetry, they were multiplied by six. Then, the indentation curves were plotted. The maximal height of the pile-up or the depth of sink-in was found on the plane of symmetry. The values from the substep at the end of the loading part, when the indentor displacement is the highest, were taken to eliminate the error caused by the residual stresses which caused the growth of the pile-up in the process of unloading.

\section{Results and discussion}

The main goal of this paper was to determine the dependency between the height of the pile-up and the depth of sink-in on the coefficient of friction (Fig. 5). The maximal pile-up and sink-in were on the plane of symmetry in the middle of the indenter side. Results from the FEM calculations showed that the depth of the sink-in is nearly independent on the friction. The height of the pile-up was strongly depended on the friction until $\mathrm{f}=0.15$ (Fig. 5). For higher values, the maximal height of the pile-up was nearly the same.

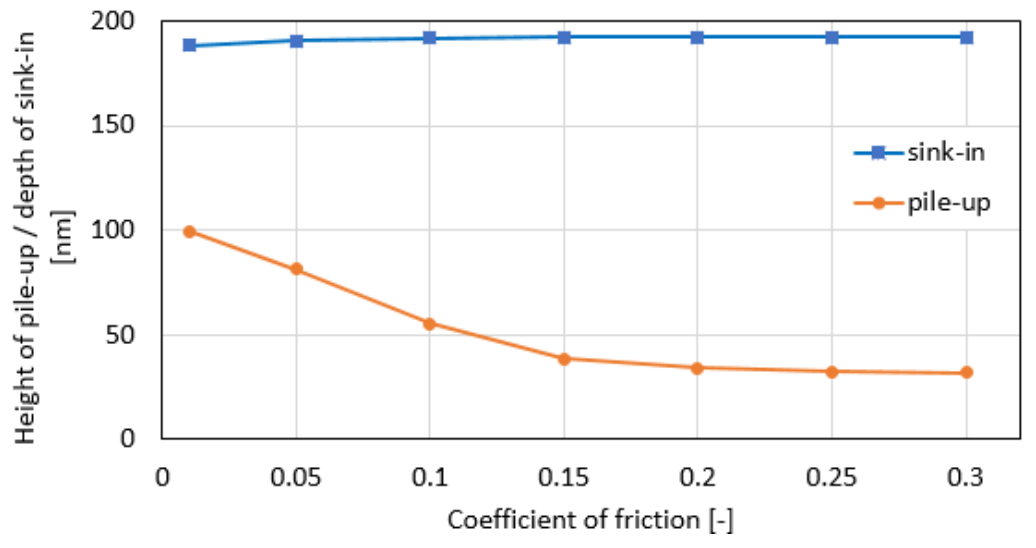

Fig. 5: The dependency of the depth of the sink-in and the height of the pile-up on the coefficient of friction.
As was expected, the coefficient of friction had an influence on the height of pile-up region. This agrees with the calculations made by Adam and Swain (2011), where the same dependency is shown. Apart from the coefficient of friction, the resultant value of the height of the pile-up was very sensitive to the accuracy of the model of material. The multilinear model is used in this paper to get accurate results.

The calculated indentation curves for steel were compared with the experimental data (Fig. 6). The FEM results showed slightly lower values of force than the experimental curve. The curves obtained by FEM are nearly identical, but the unloading parts of these curves are very slightly less steep for the bigger values of the friction. This behavior corresponds to Adam and Swain's paper (2011), but the curves there are calculated for much more compliant material, whose behavior is much more sensitive to these changes. 
Although there was a difference between the maximal values of the forces obtained by the calculations and the experiment, the calculated curves had expected shapes. The difference between the results could be caused by the behavior of the material, which is difficult to calculate more precisely with commonly used material models. The employment of more complex material models should make the results more accurate and will be done in the future work.

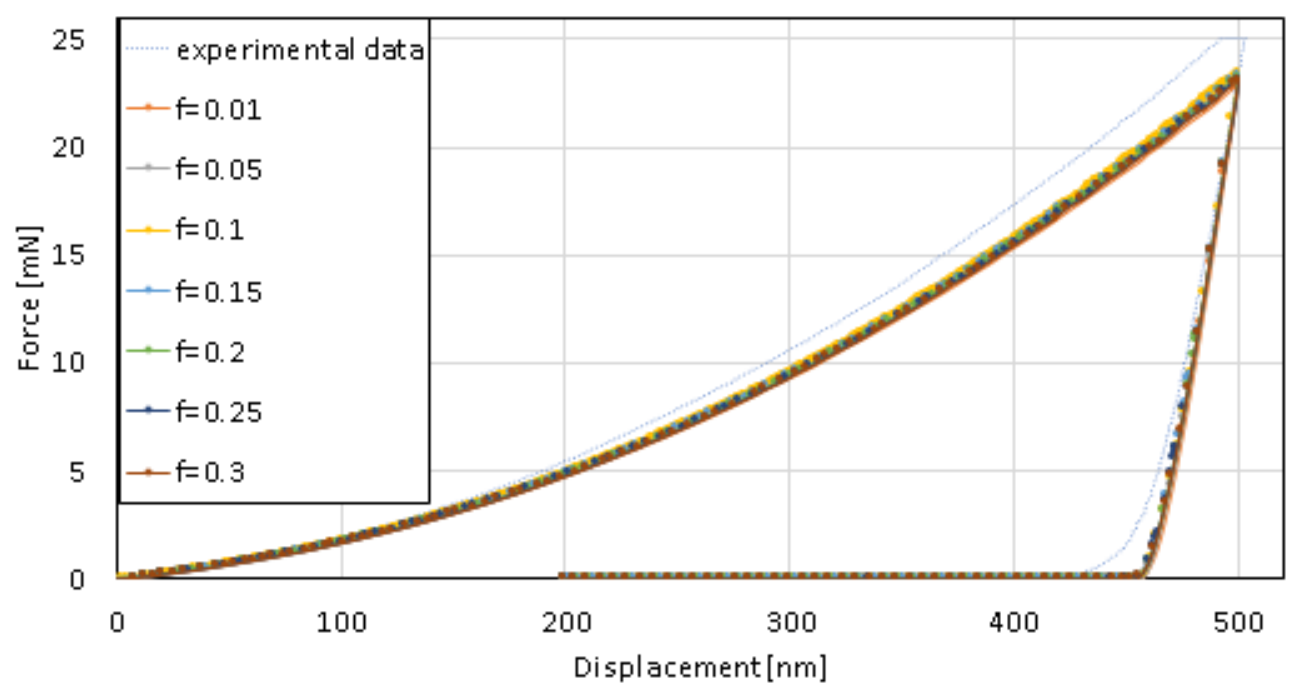

Fig. 6: Experimental and calculated indentation curves.

\section{Conclusion}

The impact of the coefficient of friction on the results of FEM calculations has been assessed. The indentation curves for different values of the coefficient of friction for two materials and the dependencies between the height of the pile-up and the depth of sink-in on the friction were

calculated. The results showed that the sink-in is nearly independed of the friction but the height of pile-up strongly decreases until the $\mathrm{f}=0.15$ and then it levels out. The coefficient of friction had only a small influence on the unloading curves, if the pile-up occurred. The determination of Young's modulus by the Oliver-Pharr analysis should not be affected by the coefficient of friction, because the difference in the steepness of the unloading curves could be corrected by the contact area which is influenced by the height of the pile-up. The calculated indentation curves showed lover values of the force than the experimental curves. It could be due to the inaccuracy of the model of material or a real shape of the indenter, where was the bluntness. To obtain more precise results the real shape of the indenter should be measured and more complex material model used. Future work should be aimed if the friction does not influence the calculated Young's modulus.

\section{Acknowledgement}

This study was realized with the support by the grant FSI-S-20-6164 and with the institutional support RVO: 61388998. In addition, this work has been supported by the project No. FV 19 - 07 funded by The Ministry of Education, Youth and Sports (MEYS, MŠMT in Czech) institutional support.

\section{References}

Adam, C. J. and Swain, M. V. (2011) The effect of friction on indenter force and pile-up in numerical simulations of bone nanoindentation [Online]. Journal of the Mechanical Behavior of Biomedical Materials, 4(7), 1554-1558.

Kovár, J. and Fuis, V. (2019) FEM simulation of the nanoindentation test with rigid and non-rigid indenter. In Engineering mechanics 2019 (eds.I. Zolotarev \& V. Radolf), pp. 189-192.

Kovár. J., Fuis, V., and Tomáštík, J. (2019) Influencing the indentation curves by the bluntness of the Berkovich indenter at the FEM modelling.

Oliver, W. C., and Pharr, G. M. (1992) An improved technique for determining hardness and elastic modulus using load and displacement sensing indentation experiments. Journal of Materials Research, 7(06), 1564-1583.

Oliver, W. C., and Pharr, G. M. (2004) Measurement of hardness and elastic modulus by instrumented indentation: Advances in understanding and refinements to methodology [Online]. Journal of Materials Research, 19(01), $3-20$. 